\title{
Molecular Recognition of Iminium Ions in Water
}

\author{
Vy M. Dong, Dorothea Fiedler, Barbara Carl, Robert G. Bergman ${ }^{\star}$, and Kenneth N. \\ Raymond \\ Department of Chemistry, University of California, Berkeley, CA 94720-1460, USA
}

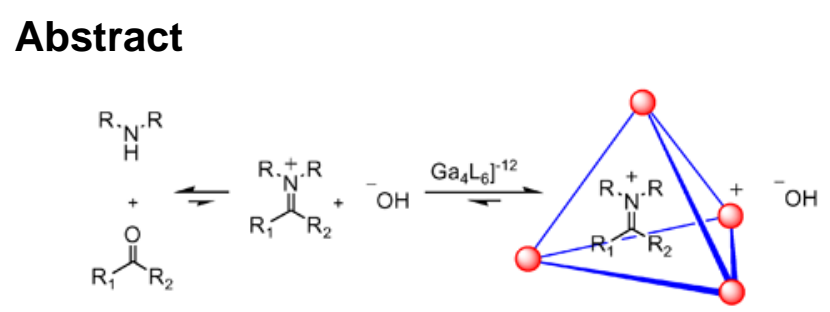

Iminium ions are known to exist only transiently in aqueous solution due to their high reactivity towards hydrolysis. In this communication, we report on the generation and stabilization of iminium ions in aqueous solution via molecular encapsulation using a $\mathrm{K}_{12} \mathrm{Ga}_{4} \mathrm{~L}_{6}$ host. Our studies revealed that tetrahedral host 1 can encapsulate a variety of iminium cations in a molecular recognition process that is selective based on the charge, hydrophobicity, size, and shape of the guest.

Iminium ions play a significant role in both biology and chemistry as key intermediates in a range of enzyme-catalyzed processes ${ }^{1}$ and synthetic transformations. ${ }^{2}$ For example, nature uses class I aldolase enzymes to catalyze an aldol process that proceeds through an iminium cation under physiological conditions. In the growing field of organocatalysis, an array of enantioselective methods has been achieved that rely on the use of chiral iminium ions. ${ }^{3}$ Methods to efficiently generate these reactive species in situ typically require acidic conditions and/or organic solvents. In aqueous solution, iminium ions exist only transiently due to their high reactivity towards hydrolysis. ${ }^{4}$ Intrigued by the ability of covalent and self-assembled hosts to sequester a number of reactive species, ${ }^{5}$ we sought to use host-guest chemistry as a novel means of accessing iminium ions. We now report the quantitative generation and stabilization of these species by encapsulation at room temperature in aqueous solution.

Raymond and coworkers have previously developed a water-soluble chiral $\left[\mathrm{Ga}_{4} \mathrm{~L}_{6}\right]^{12-}$ tetrahedral assembly 1 which is self-assembled from simple metal and ligand components. ${ }^{6}$ In this assembly, four metal atoms are located on the vertices and bridged by six bis-bidentate catecholamide ligands (Figure 1). The hydrophobic pocket of this assembly has a strong propensity for binding cationic guests (e.g. alkylammonium cations and organometallic complexes) ${ }^{7}$ and stabilizing reactive phosphonium, ${ }^{8}$ diazonium, ${ }^{9}$ and organometallic intermediates. ${ }^{10}$ While some of these species are rendered inert for extended periods, others exhibit unusual cavity-controlled reactivity.

Based on the affinity of nanovessel 1 for cationic species, we envisioned that this assembly could favor the encapsulation of iminium ions generated in situ from amines and ketones in water (Scheme 1). The concentration of an iminium ion is negligible in aqueous solution at neutral or basic $\mathrm{pH}$. However, we expected this unfavorable equilibrium could be dramatically

E-mail: rbergman@ berkeley.edu . 
altered by molecular encapsulation. If possible, this would represent a unique strategy for generating iminium ions inside the chiral pocket of a water-soluble nanovessel.

In agreement with our hypothesis, combination of pyrrolidine and acetone in an aqueous solution containing 1 resulted in formation of the desired inclusion-complex $[2 \subset 1]^{11-}$ (Table 1, entry 1). In the absence of host $\mathbf{1}$, no iminium ion could be observed by ${ }^{1} \mathrm{H}$ NMR analysis.

A wide range of ketones (from acetone to 2-nonanone) are tolerated in this process with binding efficiencies that varied according to the size of the guest (Table 1). ${ }^{11}$ In general, the hydrophobicity of the iminium ion (and thus its affinity for the hydrophobic host pocket) is expected to increase with the number of carbons in its alkyl chain. ${ }^{12}$ However, this affinity is compromised when the size of the guest becomes too large for the cavity. For 2-ketones, the optimal fit for the hydrophobic pocket was observed with pyrrolidinium ions $\mathbf{4}$ and $\mathbf{5}$ derived from 2-pentanone and 2-hexanone (entry 3 and 4). Iminium cations generated from smaller ketones (i.e. acetone and 2-butanone) were encapsulated with lower efficiencies (entries 1 and 2). No iminium ions could be observed using 2-undecanone as this guest is too large to fit inside the host cavity (entry 8). A similar trend in binding efficiency versus alkyl chain length was observed for pyrrolidinium guests derived from a series of 3-ketones (see entries 9-13).

The ${ }^{1} \mathrm{H}$ NMR resonances for the iminium guest appear in a characteristically upfield region of the spectrum ( 0.5 to $-2.0 \mathrm{ppm})$ due to shielding by the aromatic walls of the host. Based on their ${ }^{1} \mathrm{H}$ NMR spectra, it appears that iminium cations $\mathbf{4}$ and $\mathbf{1 1}$ are encapsulated with their alkyl chains in a fully extended conformation. ${ }^{13}$ As gauche interactions are minimized, such extended conformations are expected to be the most energetically favorable. Of note, guests 4 and 11 exhibit the highest relative binding affinities. However, spectra for cations larger than $\mathbf{4}$ or $\mathbf{1 1}$ show that these alkyl chains exist in a coiled (rather than extended) conformation. As such, relatively large iminium ions are accommodated by packing their alkyl chains within the binding pocket. ${ }^{11}$ Our observation of this supramolecular coiling effect is consistent with Rebek's recent reports for the helical coiling of alkanes inside a cavitand. ${ }^{14}$.

The molecular recognition of iminium ions by tetrahedral assembly $\mathbf{1}$ was found to be highly selective based on the structure of the amine component. In addition to pyrrolidine, azetidine can be used to form the corresponding iminium ion-inclusion complexes with a large range of ketones. However, iminium ions derived from other cyclic amines, including aziridine, piperidine and morpholine, were not encapsulated to any observable extent. Primary amines, including ethylamine, propylamine, and butylamine could be used to generate weakly bound iminium ions. ${ }^{15}$.

Competition experiments conceptually analogous to those reported by Lehn ${ }^{16}$ were conducted to further study the ability of host $\mathbf{1}$ to bind iminium ions. Lehn and coworkers demonstrated that the enzyme carbonic anhydrase could selectively bind imines generated from a dynamic combinatorial library of aldehydes and amines. Could synthetic host $\mathbf{1}$ similarly bind an iminium cation generated from a mixture of amines and ketones in water? To investigate this question, a competition experiment was conducted by treating a mixture of pyrrolidine, azetidine, and butylamine to an aqueous solution of 2-heptanone and host $\mathbf{1}$ (Scheme 2). Remarkably, host 1 preferentially binds to the azetidinium ion; the ratio of azetidiniumcomplex 15 to pyrrolidinium-complex 6 was 74:26 based on ${ }^{1} \mathrm{H}$ NMR analysis. Iminium ions derived from butylamine could not be observed under these conditions.

A second competition experiment involving treatment of azetidine and assembly $\mathbf{1}$ with a set of three differentially substituted heptanones resulted in the selective formation of the azetidium-complex 17 derived from 4-heptanone (74\%) (Scheme 3). The results of these 
competition studies highlight an enzyme-like ability of synthetic host $\mathbf{1}$ to recognize subtle structural variations in the guest cation.

In summary, our studies revealed that $\left[\mathrm{Ga}_{4} \mathrm{~L}_{6}\right]^{12-}$ host $\mathbf{1}$ can encapsulate a variety of iminium cations in a molecular recognition process that is selective based on the charge, hydrophobicity, size, and shape of the guest. In addition, we have demonstrated a novel use of host-guest chemistry to generate iminium ions under unconventional conditions - in water at basic $\mathrm{pH}$. Once encapsulated the iminium ions remained stable for months at room temperature. Current efforts are focused on identifying reagents that will allow further elaboration of the encapsulated guest. In addition, this strategy will be used to access other cationic reactive intermediates and develop new transformations in aqueous solution.

\section{Supplementary Material}

Refer to Web version on PubMed Central for supplementary material.

\section{Acknowledgment}

This work was supported by the Director, Office of Energy Research, Office of Basic Energy Sciences, Chemical Sciences Division, of the U.S. Department of Energy under Contract DE-AC02-05CH11231. VMD and BC gratefully acknowledge postdoctoral fellowships from the NIH and DFG, respectively.

\section{References}

1. Wong, CH.; Whitesides, GM. Enzymes in Synthetic Organic Chemistry, Pergamon. Oxford: 1994. Jencks, WP. Catalysis in Chemistry and Enzymology. New York: Dover; 1987.

2. Bohme, H.; Haake, M. Iminium Salts in Organic Chemistry; Advances in Organic Chemistry. Taylor, EC., editor. New York: John Wiley and Sons; 1976. p. 145

3. Berkessel, A.; Gröger, H. Asymmetric Organocatalysis: From Biomimetic Concepts to Applications in Asymmetric Synthesis. Wiley-VCH; 2005.

4. Eldin S, Digits JA, Huang ST, Jencks WP. J. Am. Chem. Soc 1995;117:6631.

5. Vriezema DM, Aragones MC, Elemans J, Cornelissen J, Rowan AE, Nolte RJM. Chem. Rev 2005;105:1445. [PubMed: 15826017]

6. Caulder DL, Bruckner C, Powers RE, Konig S, Parac TN, Leary JA, Raymond KN. J. Am. Chem. Soc 2001;123:8923. [PubMed: 11552799]

7. Fiedler D, Leung DH, Bergman RG, Raymond KN. Accounts Chem. Res 2005;38:349.

8. Ziegler M, Brumaghim JL, Raymond KN. Angew. Chem., Int. Ed 2000;39:4119. (b) Brumaghim JL, Michels M, Raymond KN. Eur. J. Org. Chem 2004;22:4552.

9. Brumaghim JL, Michels M, Pagliero D, Raymond KN. Eur. J. Org. Chem 2004;24:5115.

10. Fiedler D, Raymond KN. Angew. Chem., Int. Ed 2006;45(5)

11. Although not shown in Table 1, branched ketones (e.g $\alpha$ - and $\beta$ - branched pentanones) will form inclusion complexes as well.

12. Parac TN, Caulder DL, Raymond KN. J. Am. Chem. Soc 1998;120:8003.

12. See supporting information (SI) for NMR spectra and more details.

13. (a) Trembleau L, Rebek J. Science 2003;301:1219. [PubMed: 12947192] (b) Purse BW, Rebek J.

Proc. Natl. Acad. Sci. U. S. A 2006;103:2530. [PubMed: 16477037]

14. Binding efficiencies were less than $10 \%$ by ${ }^{1} \mathrm{H}$ NMR integration.

15. Huc I, Lehn JM. Proc. Natl. Acad. Sci. U. S. A 1997;94:2106. [PubMed: 9122156] 

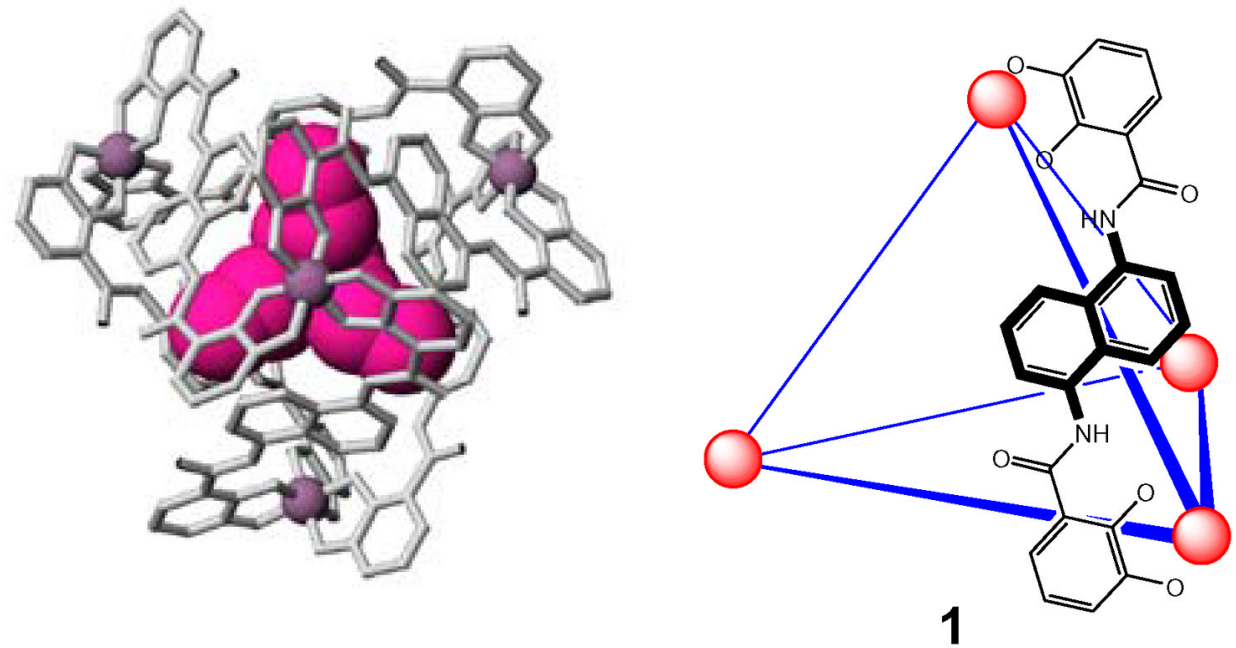

Figure 1.

(Left) CAChe model of $\left[\mathrm{Pr}_{4} \mathrm{~N}^{+} \subset \mathrm{Ga}_{4} \mathrm{~L}_{6}\right]^{11-}$ assembly; showing the $\Delta, \Delta, \Delta, \Delta$ isomer. (Right) General schematic of the $\left[\mathrm{Ga}_{4} \mathrm{~L}_{6}\right]^{12-}$ tetrahedron. One ligand is drawn while the additional ligands are represented by lines. 


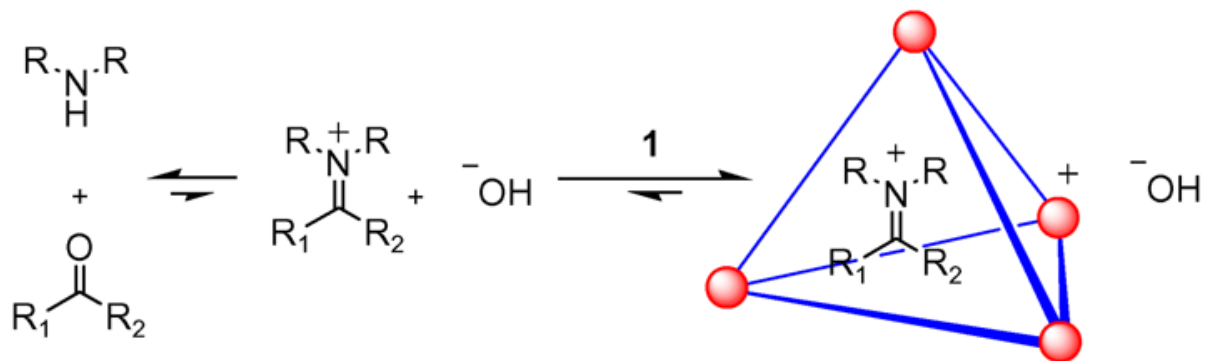

Scheme 1.

Stabilization of iminium ions in aqueous solution by molecular encapsulation. 

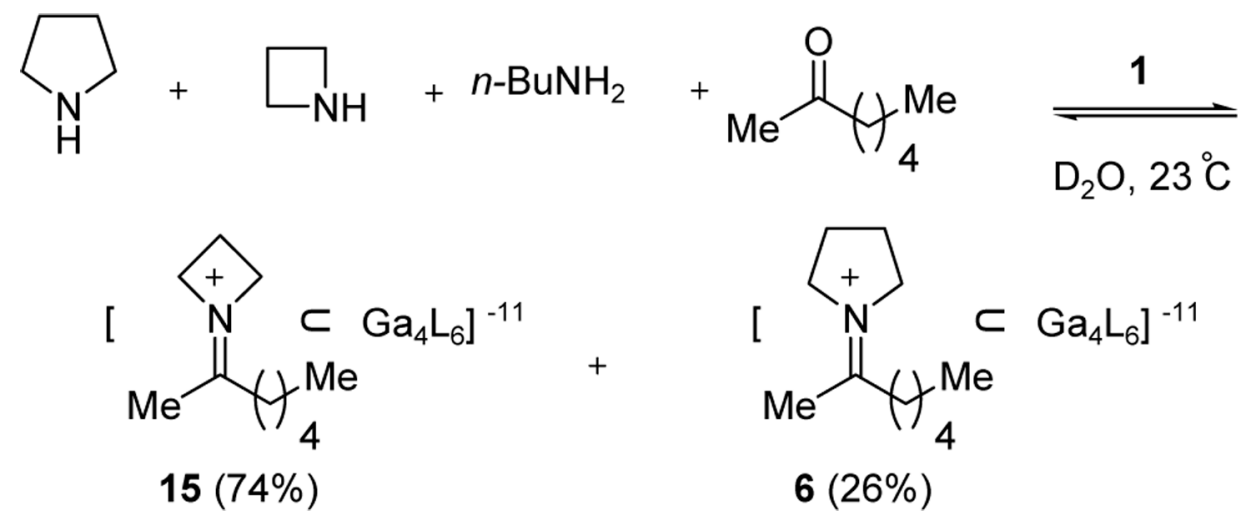

Scheme 2.

Competition experiment between amines. 
<smiles>CCC(=O)C(C)C(C)C(=O)C(C)CC(=O)C(C)C</smiles><smiles></smiles>

$15(18 \%)$<smiles></smiles>

$16(8 \%)$<smiles>CCCCC(C)C(C)C(C)C</smiles>

$17(74 \%)$

Scheme 3.

Competition experiment between 2-, 3-, and 4-heptanone. 


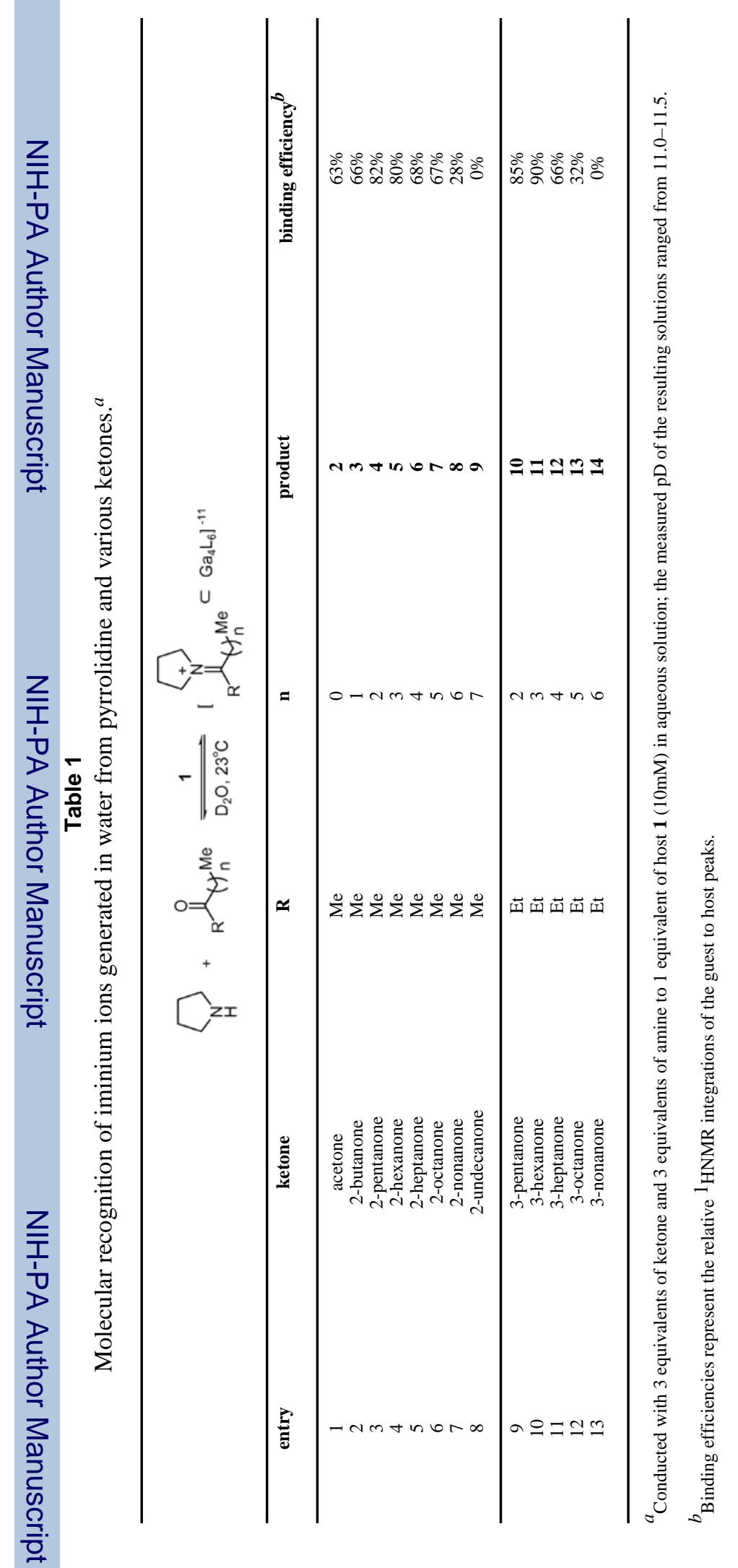

